\title{
Nuclear adiabatic effects of electron-positron pairs on the dynamical instability of very-massive stars
}

\author{
L. Garba ${ }^{a}$ and F. A. Ahmed ${ }^{b}$ \\ ${ }^{a}$ Department of Physics, Faculty of Science, Yusuf Maitama \\ Sule University, 3220 Kano, Nigeria. \\ ${ }^{b}$ Department of Physics, Faculty of Science, University of \\ Diyala, Diyala, Iraq. \\ *e-mail: ridwangy@yahoo.com
}

Received 1 June 2021; accepted 4 August 2021

\begin{abstract}
The adiabatic effects of electron-positron pair-production on the dynamical instability of very-massive stars is investigated from stellar progenitors of carbon-oxygen cores within the range of $64 \mathrm{M}_{\odot}<\mathrm{M}_{\mathrm{CO}}<133 \mathrm{M}_{\odot}$ both with and without rotation. At a very high temperature and relatively low density, the production of electron-positron pairs in the centres of massive stars leads the adiabatic index below 4/3. The adiabatic quantities are evaluated by constructing a model into a thermodynamically consistent electron-positron equation of state $(\mathrm{EoS})$ table. It is observed that the adiabatic indices in the instability regions of the rotating models are fundamentally positive with central temperature and density. Similarly, the mass of the oxygen core within the instability region accelerates the adiabatic indices in order to compress the star, while the mass loss and adiabatic index in the non-rotating model exponentially decay. In the rotating models, a small amount of heat is required to increase the central temperature for the end fate of the massive stars. The dynamics of most of the adiabatic quantities shosw a similar pattern for all the rotating models. The non-rotating model may not be suitable for inducing instability. Many adiabatic quantities have shown great effects on the dynamical instability of the massive stars due to electron-positron pair-production in their centres. The results in this work would be useful for better understanding of the end fate of very-massive stars.
\end{abstract}

Keywords: Massive stars; pair-production; stellar instability.

DOI: https://doi.org/10.31349/RevMexFis.68.011403

\section{Introduction}

In a general term, the stability and/or instability of a star is determined by the magnitude of its adiabatic index $\gamma_{a d}[1]$, being a star dynamically unstable when the ratio of its adiabatic index is less than 4/3 [2]. In 1990, Kippenhahn, Weigert and Weiss [3], emphasized that due to hydrodynamic instability, the radial structure of stars is adiabatic dependent. Whence, the adiabatic properties are greatly important in massive objects such as stars and in many astrophysics and plasma research [4]. However, Pols [5], confirmed that pair-production leads to low adiabatic index which triggers explosion of massive stars as Pair-Instability Supernova (PISN). Models of very-massive stellar evolution may provide qualitative information about the instability of PISN progenitor stars. For this reason, there is the need to identify the effects of adiabatic quantities in any particular stellar model. A stellar model may not be beautiful if its stability and/or instability is unknown. This is why many cases were considered and the stability of many models was investigated. Consequently, the effects of adiabatic changes due to pair-production in massive stars, appeared worth-while to be examined and studied, considering its role in the stability and/or instability, collapse, and explosion of massive stars. For this reason, the discovery of a super-luminous supernova (SLSN), has rapidly increased the interest in pair-instability explosion of massive stars. Nuclear adiabatic process, considered in this work, can be defined as a thermonuclear process which is characterized by a rapid change of state without heat exchange between the system and its surroundings [4].

In massive stars, however, electron-positron pairs are produced as a result of high energy photons in their core [1,613]. And the energy needed to create the rest mass of these pairs reduce the adiabatic index to less than 4/3 [11], as mentioned earlier. Meanwhile, the different magnitude of the adiabatic index could make the star unstable, or, in general terms, term, the stability of a star is affected by the decrease in its adiabatic index [2]. Once any collapsing star enters the electron-positron pair-production region (Fig. 4), it becomes destabilized by the pair-production through a decrease in the adiabatic index [14]. One significant role of pairproduction (inside the core of massive stars) is its effect on the instability and subsequent explosion of the stars, which occur during the advanced nuclear burning stages of evolution. In general, when the abundance of degenerate electrons and positrons in centres of massive stars are comparable to each other, there exists a region (Fig. 4) in the temperaturedensity plane where the adiabatic index is lower than 1.33. The adiabatic exponents and their derivatives are, therefore, a major event in stellar evolution, ranging from the stellar formation, pulsation, convection to core collapse and supernova explosion. In particular, the adiabatic derivatives (exponent and temperature gradient) measure the thermodynamic response such as the expansion and/or compression, charac- 
terized by dynamic instability as well as convection instability of stars $[3,15,16]$. Similarly, the behaviour of a star after it is adiabatically expanded or compressed depends on the numerical value of the adiabatic index $\left(\gamma_{a d}\right)$. Thus, we know that the star's internal energy derivatives with respect to pressure, temperature and density are relevant for its stability against convection and dynamical motion. The initial mass of a star candidate to experience this pair-production instability is a subject of debate. Woosley, Heger and Weaver [17], stressed that a star with initial mass from around 120 $\mathrm{M}_{\odot}$ and higher at high temperature and density would create electron-positron pairs and cause instability in their cores. While Chatzopoulos and Wheeler [18], investigated the minimum mass of a main-sequence star capable to encounter the instability region. This latter work suggested that the ability of a star to create electron-positron pairs and induce the instability is controlled by its oxygen core mass, which in turn. In main-sequence stars depend on mass loss, metallicity, and rotationally induced mixing as well as convective and semiconvective instability. However, this was long ago predicted by Barkat, Rakavy and Sack [13]. It was found that stars with massive oxygen cores greater than $60 \mathrm{M}_{\odot}$ become dynamically unstable due to pair-production with the instability set in when the central temperature is high. On the metallicity effects, Heger, Fryer, Woosley, Langer and Hartmann [19] predicted that massive stars explode to PISNe within a given metallicity threshold due to strong metallicity dependence on the stellar wind. However, this metallicity threshold was later investigated in [20] and it was argued that higher metallicity models give low oxygen cores and the stars avoid the instability region [18,21,22]. Models at solar metallicity are very likely to avoid the instability region due to high metallicity [17,23] and wind mass-loss domination [22]. On the contrary, low metallicity reduces the mass loss such that the main-sequence stars could be able to encounter the instability [18]. Similarly, the effects of rotation are manifested on the helium core in the main-sequence. The mass of the helium core increases with respect to rotation which also affects the explosion mechanism through the decrease in the pairproduction threshold [24]. This led to further investigation of the helium core mass necessary for the explosion of massive stars as PISN. Heger and Woosley (2002) stressed that the helium core mass should be from around $64-133 \mathrm{M}_{\odot}$. Meanwhile, in the detailed computation of stellar models by Yusof et al [23], several factors were taken into consideration and predictions were made such that massive star progenitors expected to explode as Pair-Instability Supernovae (PISNe) were found to be between 100 and $290 \mathrm{M}_{\odot}$ rotating models in Small Magellanic Cloud (SMC) and above $450 \mathrm{M}_{\odot}$ rotating models and almost $300 \mathrm{M}_{\odot}$ non-rotating models in Large Magellanic Cloud (LMC). These progenitors are expected to keep a very high mass in order to maintain their helium core mass greater than $\sim 65 \mathrm{M}_{\odot}$. This work studies the role of the adiabatic processes inside the region where electron-positron pairs are created in the centres of massive stars, which is originated at high central temperature and relatively low density [25]. The adiabatic quantities due to the pair-production in $150 \mathrm{M}_{\odot}$ and $200 \mathrm{M}_{\odot}$ rotating models at Small Magellanic Cloud (SMC) and rotating and non-rotating $500 \mathrm{M}_{\odot}$ models at Low Magellanic Cloud (LMC), are numerically evaluated and analysed.

This paper is organized as follows: in Sec. 2, we describe the method of the model constructed and numerical input Physics. In Sec. 3 the results of the pair-production adiabatic quantities affecting the massive stars are presented. Finally, Sec. 4 is devoted to summary and conclusion.

\section{Methodology and input physical parame- ters}

The progenitor models are taken from the published grids of very massive stellar models computed with a Hydrostatic Geneva stellar evolution code using both rotation and nonrotation effects with Solar Metallicity (SM), Small Magellanic Cloud (SMC) and Large Magellanic Cloud (LMC) [23]. The Geneva code (GENEC) has been used for most massive stars observed today and in recent developments. It includes the prescription for rotation and magnetic fields in it [26]. However, the results of those grids predicted that stellar models for $150 \mathrm{M}_{\odot}$ and $200 \mathrm{M}_{\odot}$ rotating at SMC and rotating and non-rotating $500 \mathrm{M}_{\odot}$ at LMC would explode as PISNe. The prediction was achieved through simulation with a one-dimensional multi-zone hydrodynamics code (KEPLER). The KEPLER code is a stellar evolution/

TABLE I. Model properties at the start of pair-production. The properties shown (from left to right) are the initial mass, rotational velocity, metellicity, age, and mass loss. The sixth column corresponds to the final mass, followed by central density, temperature, ZAMS effective temperarure, and luminosity. The rest of the columns are the major contributor of the chemical abundance in central mass fractions from which the pairs are created. Another chemical abundance of less impact is omitted in this table, only for the purpose of simple tabulation.

\begin{tabular}{|c|c|c|c|c|c|c|c|c|c|c|c|c|c|c|}
\hline $\begin{array}{c}\mathrm{M} \\
{\left[\mathrm{M}_{\odot}\right]}\end{array}$ & $\begin{array}{c}\mathrm{V}_{e q} \\
\mathrm{Kms}^{-1}\end{array}$ & $\begin{array}{l}\mathrm{Z}_{\text {ini }} \\
{\left[\mathrm{Z}_{\odot}\right]}\end{array}$ & $\begin{array}{c}\text { Age } \\
\times 10^{6}(\mathrm{yr}) \\
\end{array}$ & $\begin{array}{c}\text { Mass } \\
\text { loss }\end{array}$ & $\begin{array}{l}\mathrm{M}_{\text {fin }} \\
{\left[\mathrm{M}_{\odot}\right]}\end{array}$ & $\begin{array}{c}\rho_{c} \\
{\left[\mathrm{~g} \mathrm{~cm}^{-3}\right]}\end{array}$ & $\begin{array}{c}\mathrm{T}_{c} \\
{[\mathrm{~K}]}\end{array}$ & $\begin{array}{r}\mathrm{T}_{\text {eff }} \\
{[\mathrm{K}]} \\
\end{array}$ & $\begin{array}{c}\mathrm{L} \\
{\left[\mathrm{L}_{\odot}\right]}\end{array}$ & $\Gamma_{\text {edd }}$ & $\begin{array}{c}{ }^{12} \mathrm{C} \\
\times 10^{-2} \\
\end{array}$ & $\begin{array}{c}{ }^{14} \mathrm{~N} \\
\times 10^{-22} \\
\end{array}$ & $\begin{array}{c}{ }^{16} \mathrm{O} \\
\times 10^{-01} \\
\end{array}$ & $\begin{array}{r}{ }^{20} \mathrm{Ne} \\
\times 10^{-02} \\
\end{array}$ \\
\hline 150 & 102.54 & 0.002 & 3.20 & -4.06 & 107 & 4.5 & 9.02 & 5.34 & 6.80 & & 6.45 & 0.00 & 8.18 & 9.57 \\
\hline 200 & 063.46 & 0.002 & 2.89 & -4.19 & 129 & 4.39 & 9.01 & 5.32 & 6.89 & 0.92 & 6.13 & 6.47 & 8.12 & 9.88 \\
\hline 500 & 000.06 & 0.006 & 2.39 & -4.22 & 75 & 4.60 & 9.01 & 5.38 & 6.63 & 0.88 & 7.62 & 0.44 & 8.19 & 8.50 \\
\hline 500 & 000.00 & 0.006 & 2.18 & -4.29 & 95 & 4.49 & 9.00 & 4.84 & 6.78 & 0.97 & 7.19 & 3.51 & 8.18 & 8.58 \\
\hline
\end{tabular}


explosion code capturing detailed treatment of nuclear burning processes incorporated with an implicit hydrodynamics. It is capable of completely studying the evolution of massive and supermassive stars, supernovae, hydrostatic and explosive nucleosynthesis [27]. Table I, summarizes the main properties for those models as induced by pair-production. Since the stellar evolution is affected by reaction rates, they are taken into consideration, using data from Nuclear Astrophysics Compilation of Reaction Rates (NACRE). All other physical ingredients used in the evolution models are described by Yusof, Hirschi, Meynet, Crowther, Ekström, Frischknecht, Georgy, Kassim and Schnurr [23].

We use an electron-positron equation of state $(\mathrm{EoS})$ table that computes the thermodynamic properties of the fundamental species such as electrons and positrons. The input of the routines are the central mass fractions of the individual compositions, the average charge per isotope and its average nucleons number, under a particular central temperature $(\mathrm{K})$ and density $\left(\mathrm{g} \mathrm{cm}^{-3}\right)$. The result of this routine gives rise to many physical properties ranging from pressure and specific thermal energies to adiabatic quantities like adiabatic index and specific heat capacities of the species involved. The equation of state chosen for this work is HELM-EoS which is developed and described by Timmes and Swesty [28]. This choice of EoS is made due to its accuracy, speed and thermodynamic consistency $[28,29]$. We modelled this EoS such that the central mass fractions of the 14 isotopes are read one after the other with their respective and individual mean nucleon number, mean charge per isotope at a particular central temperature and density as input quantities in the EoS routines.

\section{Results and discussion}

In Table I, the Eddington limit $\left(\Gamma_{E d d}\right)$ in the instability region of all the stellar models is within its theoretical limit at which

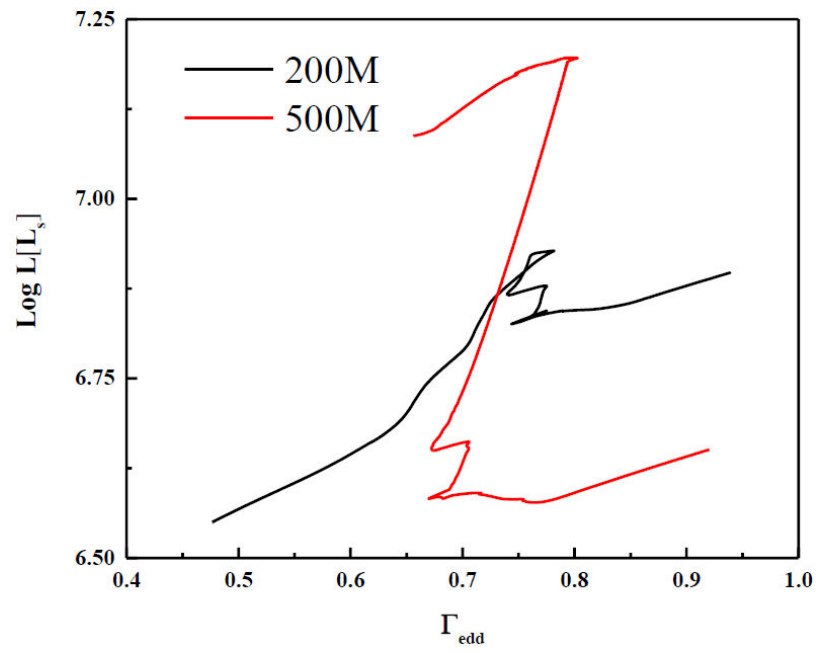

FIGURE 1. Eddington parameter for rotating $200 \mathrm{M}_{\odot}$ and $500 \mathrm{M}_{\odot}$ models with luminosity in $\mathrm{L}_{\odot}$. M represents $\mathrm{M}_{\odot}$. Data taken from [39].

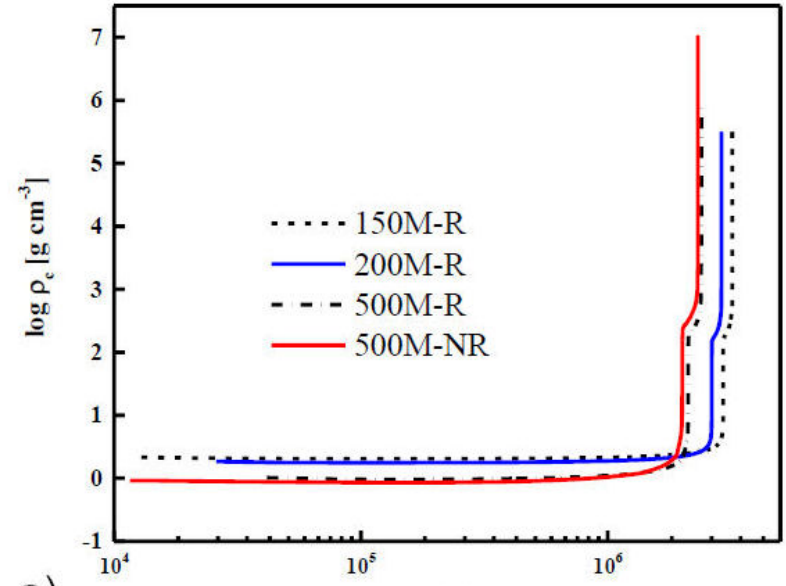

a)

$\mathbf{t}[\mathbf{y r}]$

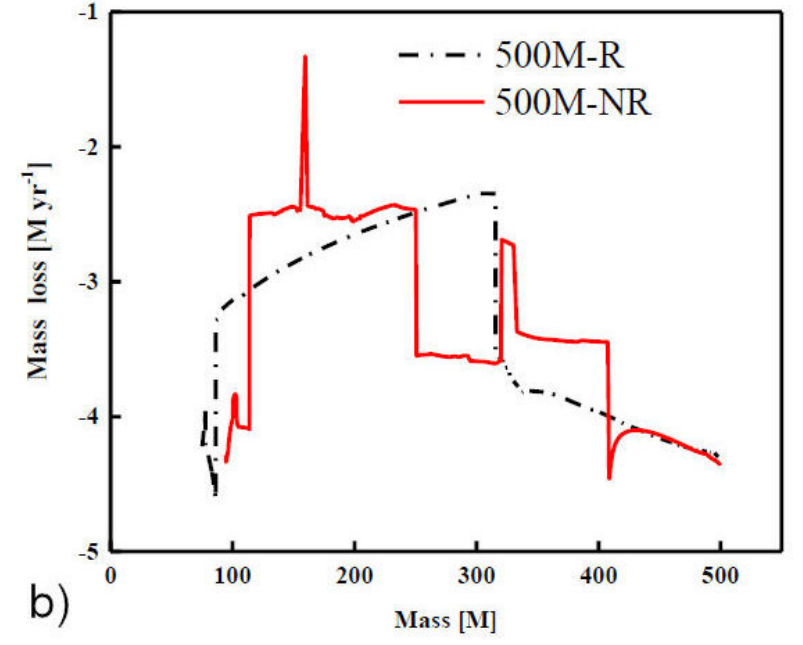

FIGURE 2. Top panel shows the time evolution of the central density with time in a year, for the models (under consideration). The dotted line is for $150 \mathrm{M}_{\odot}$, blue line for $200 \mathrm{M}_{\odot}$. And the down panel displays the mass loss rate in $\mathrm{M}_{\odot} / \mathrm{yr}$ against the final mass for $500 \mathrm{M}_{\odot}$ rotating (dash-dotted line) and non-rotating (red) models. The $\mathrm{M}$ in the graph represents solar mass $\mathrm{M}_{\odot}$ while $\mathrm{R} \& \mathrm{NR}$ represents rotating and non-rotating models respectively. The rotation reduces the mass loss and by implication the final mass.

the radiation pressure of the photon-emitting star would exceed its gravitational attraction. The maximum Eddington recorded for the 150 and $200 \mathrm{M}_{\odot}$ rotating models at SMC and rotating and non-rotating $500 \mathrm{M}_{\odot}$ models at LMC are $0.92,0.94,0.92$ and 0.98 , respectively.

There are several factors that affect these values, for example, in Fig. 1 the Eddington factor in rotating models depends on the metallicity of the star such that increasing the metallicity reduces the $\Gamma_{E d d}$, inducing the low metallicity models to easily become unstable. Other factors that affect the Eddington parameters are the rotation and mass loss. The effects of rotation can be seen from the comparison between rotating and non-rotating models. The rotating models live longer than the non-rotating ones, and the decrease in the rate of mass loss due to the rotation reduces the final mass of the star. We can see this effect in Fig. 2. Where the mass loss is 

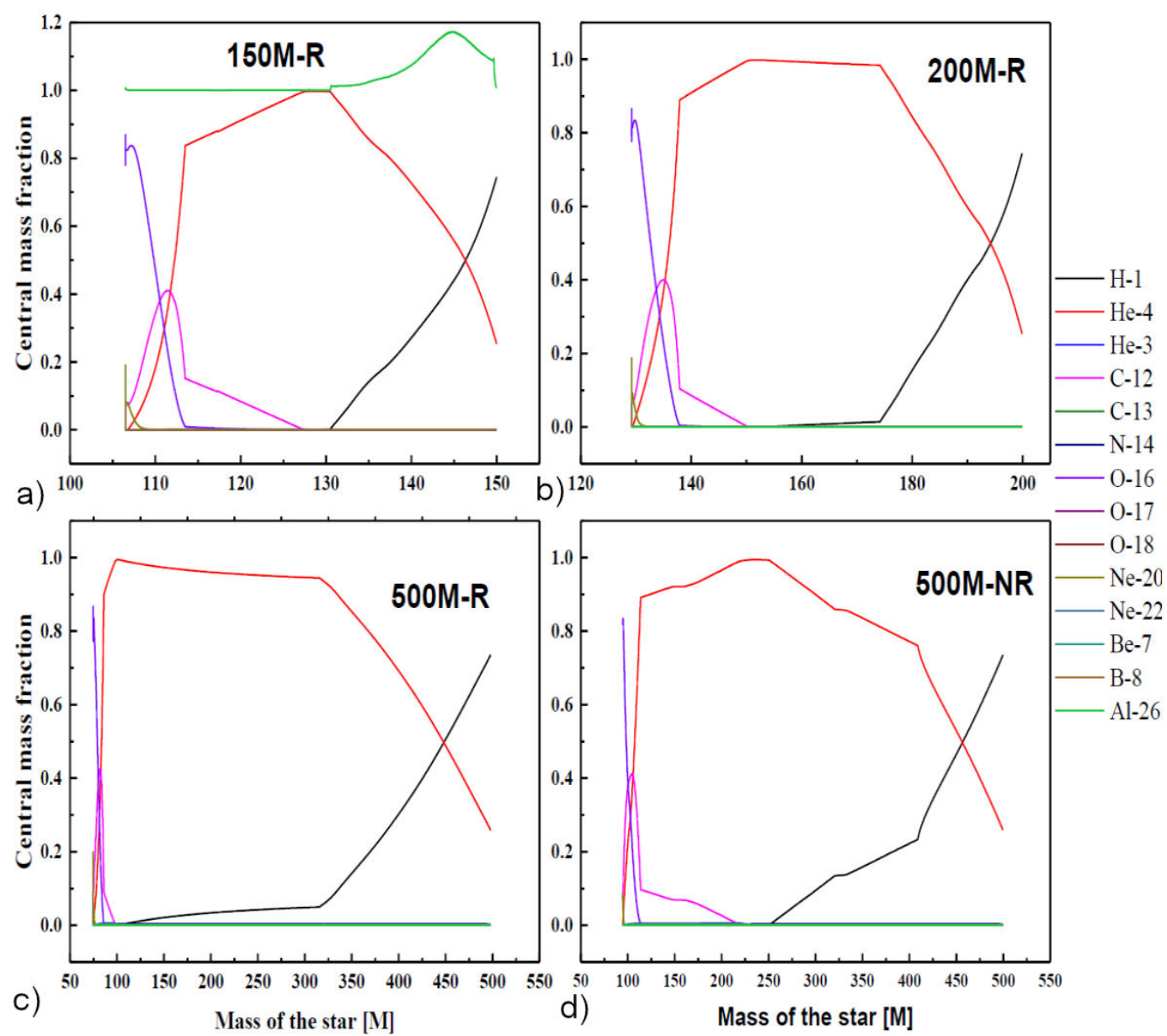

FIGURE 3. Chemical abundance within the center of $150 \mathrm{M}_{\odot}$ (up-left), $200 \mathrm{M}_{\odot}$ (up-right), rotating models at SMC and rotating (down-left) and non-rotating (down-right) $500 \mathrm{M}_{\odot}$ models at LMC. Many of the isotopes are used off for the $\mathrm{e} \pm$ thermonuclear energy production within the instability region.

plotted against the final mass for $500 \mathrm{M}_{\odot}$ rotating (red) and non-rotating (black) models at the LMC. The central abundances with respect to total mass are shown in Fig. 3. This figure clearly shows the available isotopes in the centres of the stars. Most of the isotopes have been burnt away before the instability region, remaining only the necessary ones for thermonuclear reactions taking place in the central region. In this work, we focus on the late stages of stellar evolution, starting from all burning beyond core hydrogen and helium burning [17]. The succeeding evolution after the helium burning is very quickly finished off by helium cores, thus, the mass loss is insignificant. Subsequently, the cores of the stars are mostly ${ }^{16} \mathrm{O}$, with some ${ }^{12} \mathrm{C}$ and ${ }^{20} \mathrm{Ne}$. The fact that the ${ }^{12} \mathrm{C}$ is extremely low and that the energy release from the ${ }^{20} \mathrm{Ne}$ burning is negligible, rendered their burning fuels have insignificant effects, and hence, the stars are fundamentally oxygen cores.

In Fig. 4, the evolution of the central density and temperature of the models is shown. The region enclosed by dashed-line represents where the adiabatic index is less than $4 / 3$. When enough mass of star lies inside this region, it be-

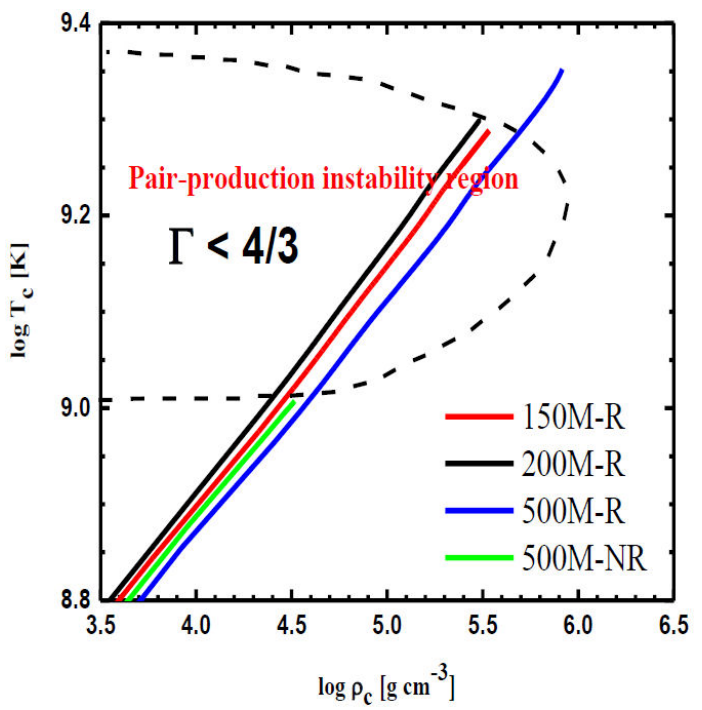

FIGURE 4. Tracks of rotating $150 \mathrm{M}_{\odot}, 200 \mathrm{M}_{\odot}$ and rotating and non-rotating $500 \mathrm{M}_{\odot}$ stelar evolution models. $\mathrm{M}$ represents the mass of a star $\left[\mathrm{M}_{\odot}\right]$ and $\mathrm{R} \& \mathrm{NR}$ are rotating and non-rotating models respectively. The models explode to PCSN model in pairproduction instability where the adiabatic index is below $4 / 3$. 


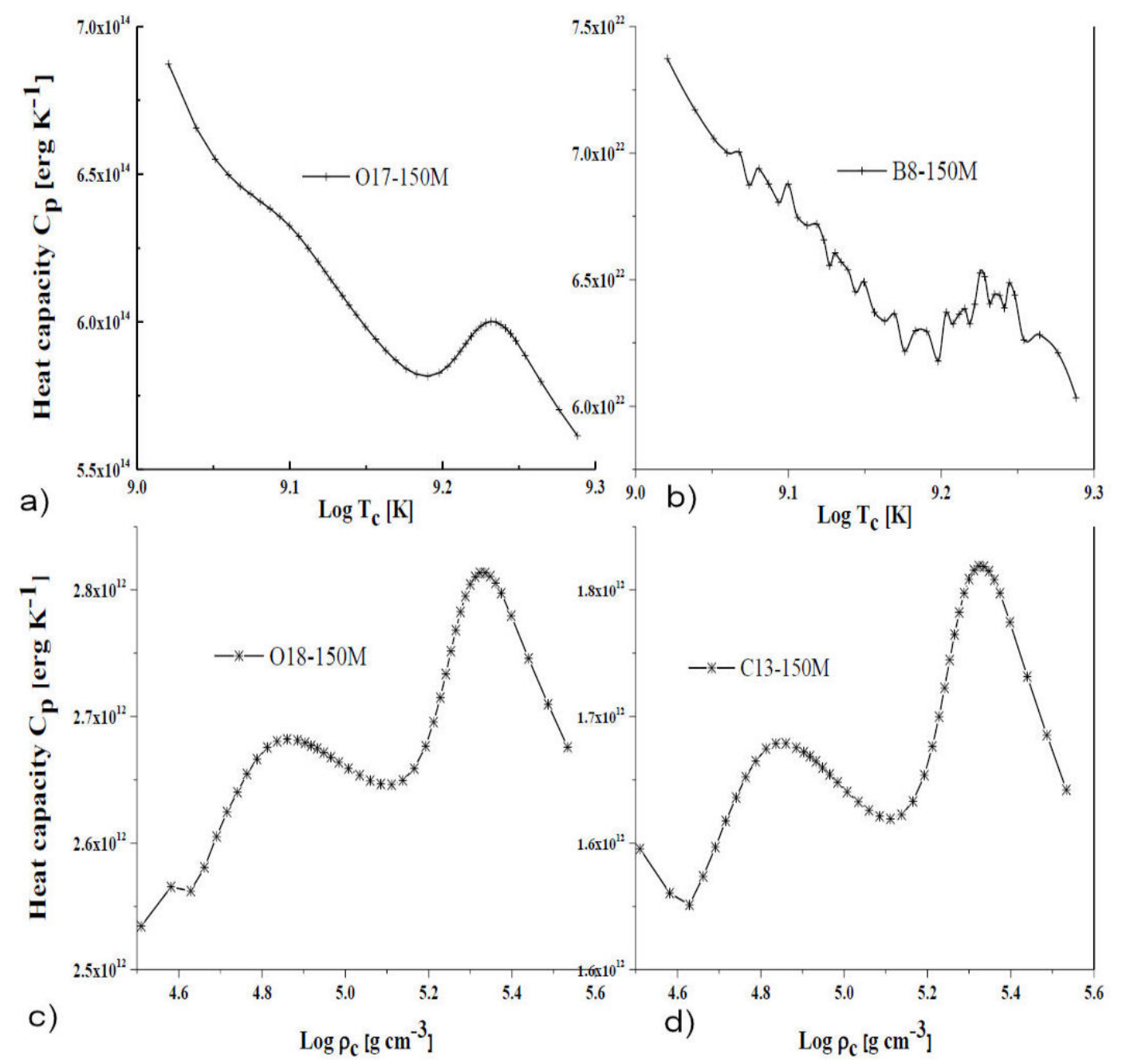

FIGURE 5. Heat capacity at constant pressure due to pair-production for the $150 \mathrm{M}_{\odot}$ progenitor model. Upper graph represents heat capacity with temperature, while the lower with density. The dynamic of the heat capacity is independent of the compositions in the instability region. The final temperature only requires a small amount of heat for the star to explode.

comes unstable. All the models under consideration became unstable and the instability proportionally increases within the pair-production region. The non-rotating model experienced the least instability by raising to a maximum central temperature $T_{c}=1.02 \times 10^{9} \mathrm{~K}$ and density $\rho_{c}=3.29 \times 10^{4}$ $\mathrm{g} \mathrm{cm}^{-3}$. On the other hand, the rotating models for $150 \mathrm{M}_{\odot}$, $200 \mathrm{M}_{\odot}$ and $500 \mathrm{M}_{\odot}$ achieved maximum central temperature and density given by $T_{c}=1.94 \times 10^{9}, 1.99 \times 10^{9}$, and $2.25 \times 10^{9}[\mathrm{~K}]$, and $\rho_{c}=3.42 \times 10^{5}, 3.02 \times 10^{5}$, and $8.25 \times 10^{5}\left[\mathrm{~g} \mathrm{~cm}^{-3}\right]$ respectively. However, the initial central temperature and density of the instability region are shown in Table I (columns 8 and 7).

In the center of massive stars, many processes occur, particularly, at a high temperature and relatively low density. One such important process is the photon disintegration into electron-positron pairs when the photon energy $(h \nu)$ is higher than the rest-mass energy of the pairs $\left(h \nu>2 m_{e} c^{2}\right)$, that only occur during the collision with a nucleus as confirmed by Pols [5]. The electron-positron pairs are created just before the formation of any element heavier than oxygen, and therefore, various physical quantities due to this pair-production in the centres of massive stars require the understanding of the pressure, specific internal energy etc., in order to describe their phenomenology. The thermodynamic coefficients that describe the pair-production process in massive stars are derived from the electron-positron EoS using thermodynamic laws, together with the internal energy equations. The most important of such thermodynamic coefficients are the specific heat the adiabatic index and the adiabatic temperature gradient [5]. In Table II we show the adiabatic quantities at the minimum and maximum central temperature obtained from the progenitor models under consideration. As we see in this table, together with Table I and Fig. 3, only advanced burning flues used in the production of the electron-positron thermonuclear energy in the instability region, show low adiabatic index below 4/3. Details of these adiabatic quantities are explained in the next sections. 

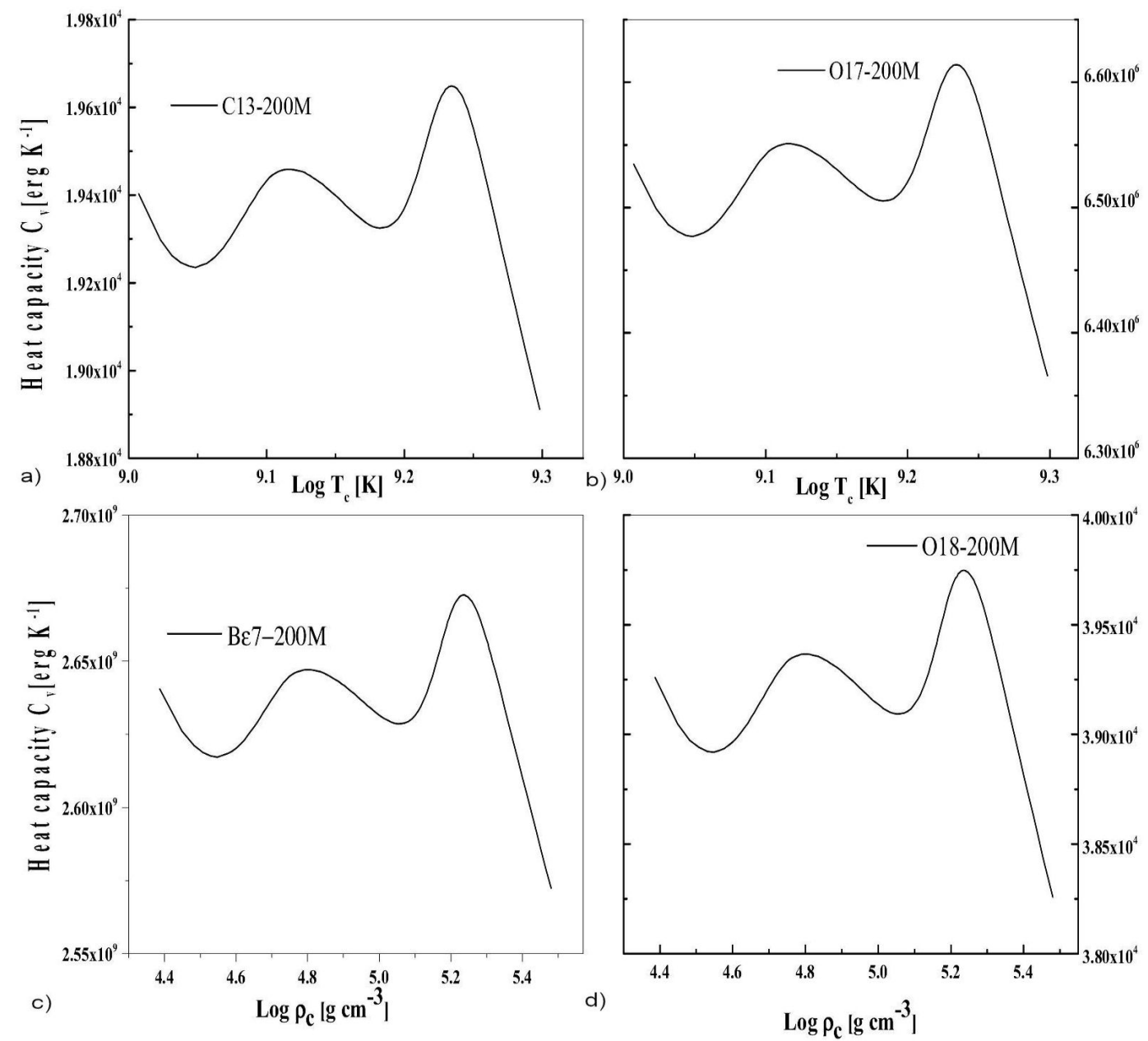

FIGURE 6 . Heat capacity at constant pressure due to pair-production for the $150 \mathrm{M} \odot$ progenitor model. Upper graph represents heat capacity with temperature, while the lower with density. The dynamic of the heat capacity is independent of the compositions in the instability region. The final temperature only requires a small amount of heat for the star to explode.

TABLE II. Adiabatic quantities at minimum and maximum temperatures in the center of massive stars with respect to selected isotopes which remain after production of electron-positron pairs. The R and NR represent rotating and non-rotating models, respectively.

\begin{tabular}{|c|c|c|c|c|c|c|c|c|c|c|c|c|}
\hline \multirow{3}{*}{$\begin{array}{l}\text { Adiabatic } \\
\text { Quantity }\end{array}$} & \multicolumn{4}{|c|}{${ }^{13} \mathrm{C}$} & \multicolumn{4}{|c|}{${ }^{18} \mathrm{O}$} & \multicolumn{4}{|c|}{${ }^{8} \mathrm{~B}$} \\
\hline & 150 & 200 & 500 & 500 & 150 & 200 & 500 & 500 & 150 & 200 & 500 & 500 \\
\hline & {$\left[\mathrm{M}_{\odot}\right]-\mathrm{R}$} & {$\left[\mathrm{M}_{\odot}\right]-\mathrm{R}$} & {$\left[\mathrm{M}_{\odot}\right]-\mathrm{R}$} & {$\left[\mathrm{M}_{\odot}\right]-\mathrm{NR}$} & {$\left[\mathrm{M}_{\odot}\right]-\mathrm{R}$} & {$\left[\mathrm{M}_{\odot}\right]-\mathrm{R}$} & {$\left[\mathrm{M}_{\odot}\right]-\mathrm{R}$} & {$\left[\mathrm{M}_{\odot}\right]-\mathrm{NR}$} & {$\left[\mathrm{M}_{\odot}\right]-\mathrm{R}$} & {$\left[\mathrm{M}_{\odot}\right]-\mathrm{R}$} & {$\left[\mathrm{M}_{\odot}\right]-\mathrm{R}$} & {$\left[\mathrm{M}_{\odot}\right]-\mathrm{NR}$} \\
\hline \multirow[t]{2}{*}{$C_{v}$} & $1.91 \mathrm{E} 4$ & $1.94 \mathrm{E} 4$ & $3.30 \mathrm{E} 03$ & $2.58 \mathrm{E} 4$ & $3.08 \mathrm{E} 4$ & $3.93 \mathrm{E} 4$ & $7.80 \mathrm{E} 3$ & $2.96 \mathrm{E} 3$ & $6.26 \mathrm{E} 14$ & $9.52 \mathrm{E} 14$ & $1.12 \mathrm{E} 15$ & $5.40 \mathrm{E} 15$ \\
\hline & $1.80 \mathrm{E} 4$ & $1.89 \mathrm{E} 4$ & $2.93 \mathrm{E} 3$ & $2.52 \mathrm{E} 4$ & $2.89 \mathrm{E} 4$ & $3.82 \mathrm{E} 4$ & $6.85 \mathrm{E} 3$ & 2.91E3 & $5.85 \mathrm{E} 14$ & $9.25 \mathrm{E} 14$ & $9.78 \mathrm{E} 14$ & $5.30 \mathrm{E} 15$ \\
\hline \multirow[t]{2}{*}{$C_{p}$} & $1.65 \mathrm{E} 12$ & $1.93 \mathrm{E} 12$ & $8.88 \mathrm{E} 10$ & $2.04 \mathrm{E} 12$ & $2.53 \mathrm{E} 12$ & $3.99 \mathrm{E} 12$ & $2.42 \mathrm{E} 11$ & $5.91 \mathrm{E} 10$ & 7.37E22 & $1.35 \mathrm{E} 23$ & $9.95 \mathrm{E} 22$ & $5.96 \mathrm{E} 23$ \\
\hline & $1.67 \mathrm{E} 12$ & $2.19 \mathrm{E} 12$ & $1.43 \mathrm{E} 11$ & $1.97 \mathrm{E} 12$ & $2.68 \mathrm{E} 12$ & $4.49 \mathrm{E} 12$ & 3.64E11 & $5.88 \mathrm{E} 10$ & $6.03 \mathrm{E} 22$ & 1.19E23 & 7.14E22 & $5.58 \mathrm{E} 23$ \\
\hline \multirow[t]{2}{*}{$\gamma_{a d}$} & 1.23 & 1.23 & 1.24 & 1.23 & 1.23 & 1.23 & 1.23 & 1.25 & 1.22 & 1.23 & 1.23 & 1.24 \\
\hline & 1.28 & 1.28 & 1.30 & 1.23 & 1.28 & 1.28 & 1.30 & 1.25 & 1.28 & 1.30 & 1.28 & 1.23 \\
\hline \multirow[t]{2}{*}{$\Delta_{a d}$} & 1.30 & 1.31 & 1.31 & 1.31 & 1.30 & 1.31 & 1.31 & 1.31 & 1.30 & 1.31 & 1.30 & 1.31 \\
\hline & 1.27 & 1.27 & 1.28 & 1.31 & 1.27 & 1.27 & 1.28 & 1.31 & 1.27 & 1.27 & 1.28 & 1.31 \\
\hline
\end{tabular}

\subsection{Heat capacities}

The heat capacities of the progenitor models are found to decrease when the central temperature and density increases within the pair-production region. Thus, the central temper- ature required for the explosion and collapse of the massive star is only achieved at a small amount of heat within the pairproduction region. In the following sections, the dynamics of heat capacity in all the considered models under consideration is discussed. 


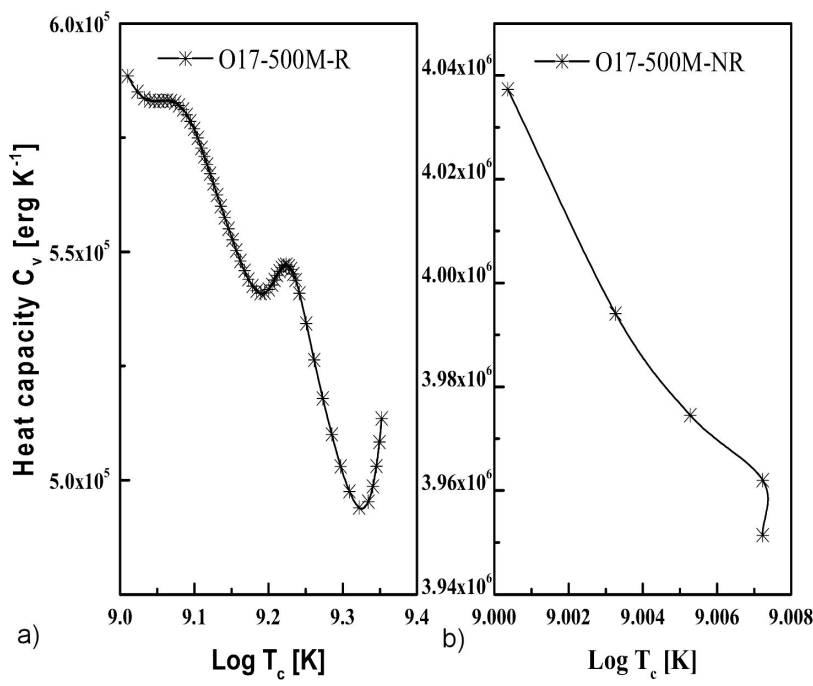

FIGURE 7. Evolution of specific heat capacity with the temperature at constant volume for rotating (left) and non-rotating (right) $500 \mathrm{M} \odot$ model. The quantity of the heat decreases by an increase in temperature of the instability region. This is also independent of the burning nucleus. The $\mathrm{M}$ represents $\mathrm{M} \odot$ while $\mathrm{R}$ and NR represent rotating and non-rotating.

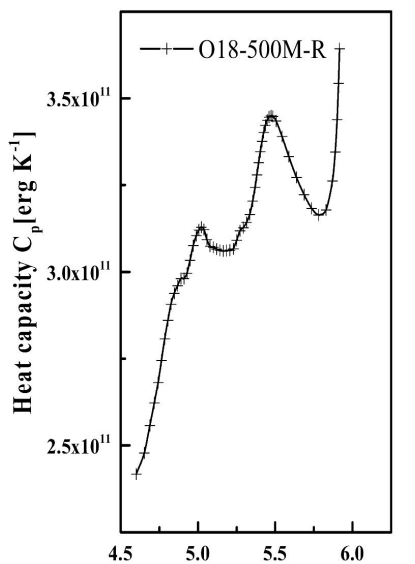

a)

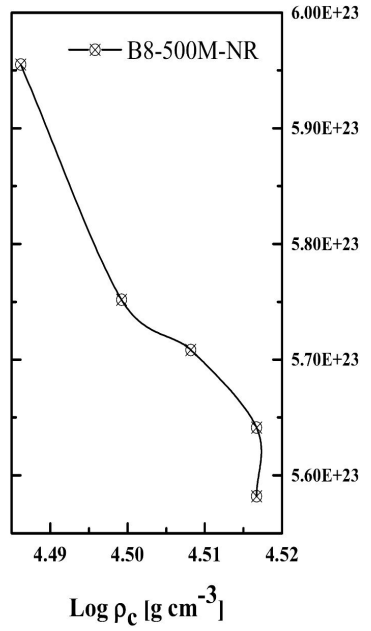

$\log \rho_{c}\left[\mathrm{~g} \mathrm{~cm}^{-3}\right]$ b)

$\log \rho_{c} \operatorname{lg~} \mathrm{cm}^{-3}$
FIGURE 8. Evolution of the specific heat capacity with density at constant pressure for rotating (left) and non-rotating (right) 500 $\mathrm{M}_{\odot}$ model. The quantity of the heat increase as the density of pairproduction rises in the rotating model while it decreases for the non-rotating model. This is uniform across the burning nuclei in the instability region. The M, R and NR same as described in Fig. 4,7 .

\subsection{1. $150 M_{\odot}$ progenitor model}

The heat capacity in the pair-instability region is independent of the composition. In Fig. 5, the behaviour of the heat capacity with respect to constant pressure in the instability region is demonstrated. At the onset of the pair-production, the compressed star speedily rise the temperature of the instability region until it starts to produce more electron-positron thermal energies. As a result of this, the region becomes completely disturbed by many thermal processes and the quantity of heat required to raise the temperature slowly reduce the density of the pair-production. The manifestation of this dynamics is that the star cools down immediately after the pair-production is ignited by subsequent neutrino energy-loss which induces the explosions.

\subsection{2. $200 M_{\odot}$ progenitor model}

While the heat capacity at constant volume shows similar dynamics for different central mass fractions, it, however, differs in magnitude. In Fig.6 the heat capacity with respect to temperature and density is shown over different central mass fractions.

However, it is observed that the quantity of heat required to raise a unit temperature necessary for the pair-production is very small. This also follows the same physical argument with the previous $150 \mathrm{M}_{\odot}$ model. The compression must have increased the pair-production energy and pressure. This thermal energy is necessary for the annihilation of the pairs. In both this model and $150 \mathrm{M}_{\odot}$ model, the rotation increases the mass loss and the mass of oxygen cores such that more pairs are produced and annihilated.

\subsection{3. $500 M_{\odot}$ progenitor model}

The heat, at constant volume and pressure, is, however, independent of the chemical abundance within the instability region, and is steadily uniform at the final mass of the explosion. In Fig. 7 and 8, the manifestation of this physical behaviour is shown. These figures compare the rotating and non-rotating models. The rotation affects the nucleosynthesis of the stellar evolution, and in the pair-production region, temperature and density play key roles in the instability of the region. The nuclear burning affects not only the heat capacity, but the rotatios as well.

In Fig. 9, it can be seen that, as the mass loss increases in the rotating models, the heat capacity slows down so that it induces the central temperature and density for the production of the pairs and subsequent annihilations and explosion of the stars. The non-rotating model experienced greater heat capacities and has the lowest mass loss which led the star further away from the pair-production region. This also confirms that the non-rotating models are not good for pairproduction, due to the fact that most of the mass of the nonrotating model is lost to heat, and therefore the star must collapse before it explodes. 

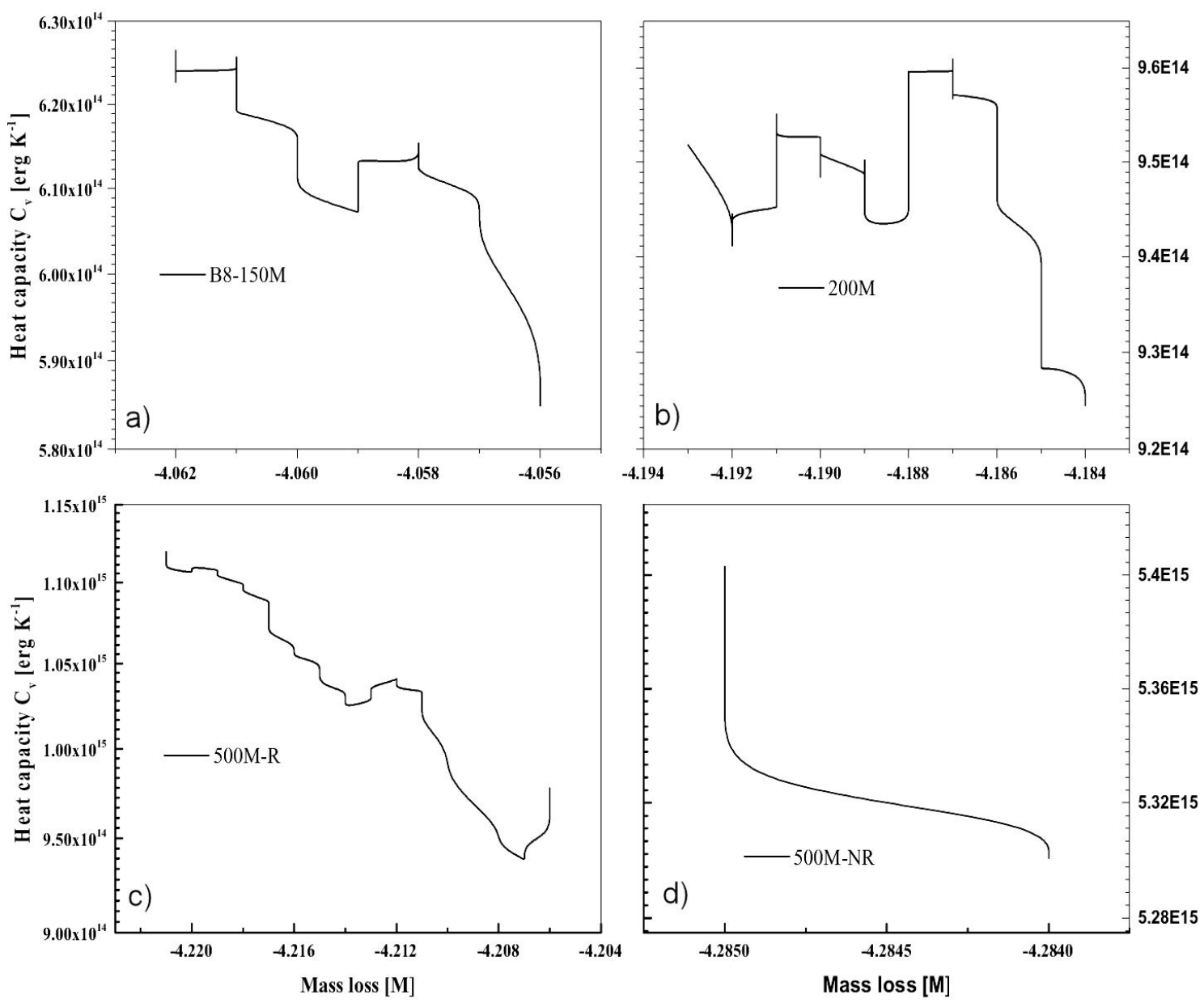

FIGURE 9. Track of heat capacities with the mass loss for all the models. The non-rotating model loses less mass and acquires higher heat capacities.

\subsection{Adiabatic index}

The adiabatic index $\gamma_{a d}=(\partial \ln P / \partial \ln \rho)_{a d}$ as a measure of pressure to adiabatic compression or expansion, is found to be proportional to the central temperature in the instability region (as in Fig. 10), and it is constant when the pressure is proportional to density (i.e. $P \alpha \rho^{\gamma_{a d}}$ ).

This adiabatic index is, however, related to dynamical stability of stars. It describes whether the star is dynamically unstable when the pressure average of $\gamma_{a d}$ is less than 4/3, [1]. The numerical values of the adiabatic indices for all the models under consideration are shown in Table II. These values show that indeed the models have adiabatic indices below 4/3 at the late burning stages for all the models. In Fig. 11, we plot the adiabatic indices with mass loss of the models. We found that the rotation reduces the mass loss and increases the adiabatic index. The non-rotating model possesses lowest adiabatic index which is the manifestation of its quick collapse as it loses most of its mass very quickly in the instability region. Similarly, the oxygen core in the rotating models induces an increase of the adiabatic indices such that the rotating stars produce electron-positron pairs.
However, the final mass of a star reduces the adiabatic index within the region such that the star could finally explode as PISN. In Fig. 10, the effects of the electron-positron pairs, in which the adiabatic indices are less than 4/3 are plotted as a function of temperature, within the instability region. in the figure, we show that the adiabatic index due to the pair production is almost independent of the nuclear burning taking place in the instability region, as predicted by Rakavy and Shaviv [12]. This adiabatic index which represents the pressure derivatives creates disturbance in the region and is fundamentally positive with temperature for all the rotating models but negatively decrease in the case of the non-rotating, as can be seen in Fig. 11. This scenario, in the rotating model, keeps the instability in the region and the star produces more pairs which later annihilate into neutrinos. Therefore, the pair production leads to a dynamical instability which is responsible for the rapid explosion of the star immediately after the production of the electron-positron pairs. Meanwhile, the sudden decrease of the adiabatic index in the non-rotating model and its quick collapse is basically due to its low mass loss and less oxygen core mass that is necessary for the pair production. 


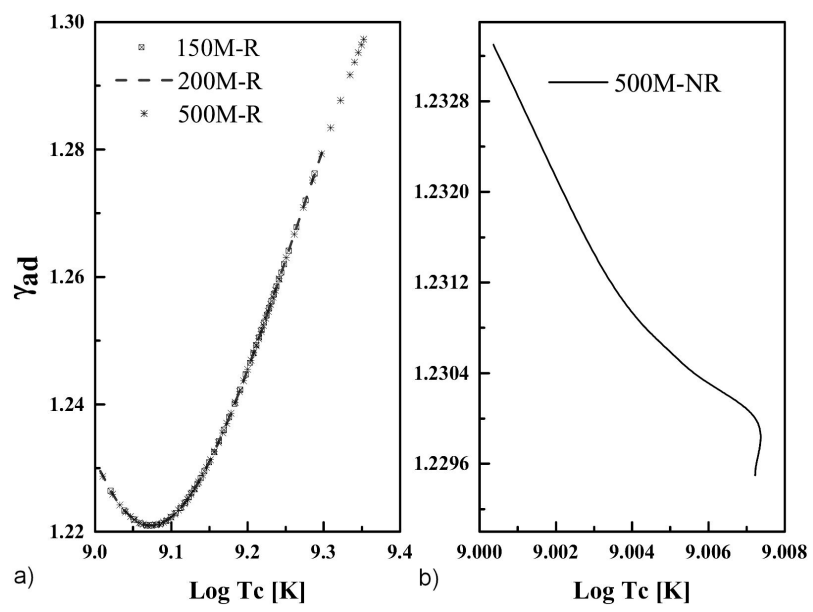

FIGURE 10. Effects of pair-production instability on the adiabatic index. The $\mathrm{M}$ represents the mass of the star in $\left[\mathrm{M}_{\odot}\right]$, while, $\mathrm{R}$ and NR stands for rotating and non-rotating models.

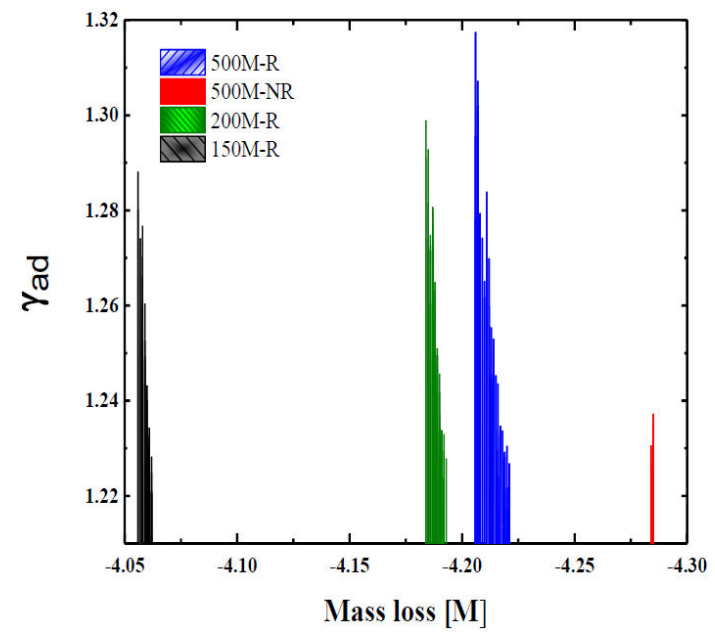

FIGURE 11. Adiabatic index with respect to a mass loss for $150 \mathrm{M}_{\odot} 200 \mathrm{M}_{\odot}$ rotating models at $\mathrm{SMC}$ and $500 \mathrm{M}_{\odot}$ rotating and non-rotating models at LMC. M, R and NR are the same as describes in Fig. 10.

\subsection{Adiabatic temperature gradients}

The temperature gradient $\nabla_{a d}=(\partial \log T / \partial \log P)_{a d}$ is another adiabatic derivative which determines the characteris- tics of the temperature under adiabatic compression or expansion of a star and is evaluated as the flow rate of energy in relation to the surface of the star. This quantity is only important for convective stability of a particular star. In Table II the minimum and maximum evaluated values of this quantity is presented in the last rows for all the models under consideration. However, we noticed equal values for all the models which clearly shows that all the models are convectively unstable and finally explode as PISNe. The convective instability, which is described by adiabatic temperature gradients, is out of the scope of this work. It is interesting to note that the adiabatic quantities show similar dynamics in all the rotating models within the pair-production regions. The non-rotating model have different pattern of instability. The quantity of heat required to rise the central temperature producing star explosion is found to have a decreasing form.

\section{Summary and conclusion}

It was predicted that 150 and $200 \mathrm{M}_{\odot}$ rotating stellar models at SMC and $500 \mathrm{M}_{\odot}$ rotating and non-rotating models at LMC are expected to explode as Pair-Instability Supernovae (PISNe). The stability and fate of these massive stars are affected by adiabatic quantities everywhere within the center of the stars. As the production of electron-positron pairs in the center of massive stars reduces the adiabatic index to less than $4 / 3$, the star becomes unstable. We have modelled a thermodynamically consistent electron-positron EoS routines and numerically evaluated the adiabatic quantities of the pair-production within the center of selected massive stars, which are expected to end as PISN. It is interesting to note that the adiabatic quantities show similar dynamics in rotating models within the instability regions. Non-rotating model possesss different pattern of instability. The amount of heat required to raise the central temperature high necessary for explosion is found to be decreasing. The significance of the adiabatic properties on the dynamical instability of the massive stars under consideration is discussed.
1. J.R. Bond, W.D. Arnett, and B.J. Carr, The Astrophyaical Journal 280 (1984) 825. https: //adsabs.harvard.edu/ pdf/1984ApJ...280..825B

2. P. Ledoux, On the dynamical stability of stars, The Astrophysical Journal 104 (1946) 333, https : / / adsabs . harvard. edu/pdf/1946ApJ...104..333L

3. R. Kippenhahn, A. Weigert, and A. Weiss, Stellar structure and evolution, (Springer, 1990), Vol. 192.

4. D. Beule, W. Ebeling, and A. Förster, Adiabatic equation of state and ionization equilibrium of dense plasma, Physica A: Statistical Mechanics and its Applications 241 (1997) 719, https://doi.org/10.1016/S0378-4371(97) 00168-4

5. O. Pols, Asrronomical Institute Uttecht (2011).

6. W. A. Fowler, The stability of supermassive stars, The Astnophysical Journal 144 (1966) 180, https: //adsabs. harvard.edu/pdf/1966ApJ...144..180F 
7. G. Rakavy and A. Shaviv, Instabilitiesin highly evolved stellar models, The Astrophysical Journal 148 (1967) 803, https://adsabs.harvard.edu/pdf/1967ApJ... $148 . .803 \mathrm{R}$

8. N. Kiriakidis, K. Fricke, and W. Glatzel, The stability of massive stars and its dependence on metallicity and opacity, MNRAS 264 (1993) 50, https://doi.org/10.1093/ mnras/264.1.50

9. G. Bisnovatyi-Kogan, Dynamic stability of compact stars, in: D. Blaschke, D. Sedrakian (eds), Superdense QCD Matter and Compact Stars NATO Science Series II: Mathematics, Physics and Chemistry 197 (2006) Springer, Dordrecht, https:// doi.org/10.1007/1-4020-3430-X01

10. K. J. Chen, MPLA 30 (2015).

11. N. Yusof, H. Abu Kassim, L. Garba, and N. Ahmad, The neutrino emission from thermal processes in very massive stars in the local universe, MNRAS 503 (2021) 5965, https : / / doi. org/10.1093/mnras/stab762.

12. N. Smith et al., SN 2006gy: Discovery of the Most Luminous Supernova Ever Recorded, Powered by the Death of an Extremely Massive Star like $\eta$ Carinae, The Astrophysical Journal 666 (2007) 1116, https://doi.org/10.1086/ 519949

13. A. Gal-Yam et al., Supernova 2007bi as a pair-instability explosion, Nature 462 (2009) 624, https://doi.org/10. $1038 /$ nature08579.

14. C.C. Joggerts and D.J. Whalen, The early evolution of primordial pair-instability supernovae, The Astrophysical Journal 728 (2011) 129, https://doi.org/10.1088/ $0004-637 \mathrm{X} / 728 / 2 / 129$

15. J. Cooke et al., Superluminous supernovae at redshifts of 2.05 and 3.90, Nature 491 (2012) 228, https://doi.org/10. $1038 /$ nature11521.

16. T. Pan, D. Kasen and A. Loeb, Pair-instability supernovae at the epoch of reionization, MNRAS 422 (2012) 2701, https: // doi.org/10.1111/j.1365-2966.2012.20837.x

17. T. Pan, A. Loeb and D. Kasen, Pair-instability supernovae via collision runaway in young dense star clusters, $M N$ RAS 423 (2012) 2203, https://doi.org/10.1111/j. 1365-2966.2012.21030.x

18. K.J. Chen, S. Woosley, A. Heger, A. Almgren and D.J. Whalen, Two-dimensional simulations of pulsational pair-instability supernovae, The Astrophysical Journal 792 (2014) 28, https: //doi.org/10.1088/0004-637X/792/1/28B

19. A. eozyrKva, 2014.

20. J. Smidt et al., Population III hipernovae, The Astrophysical Journal 799 (2015) 18, https : / doi.org/10.1088/ 0004-637X/797/2/97

21. E. Chatzopoulos et al., Emission from pair-instability supernovae with rotation, The Astrophysical Journal 799 (2015) 18, https://doi.org/10.1088/0004-637X/ $799 / 1 / 18$

22. K. Belczynski et al., The effect of pair-instability mass loss on black-hole mergers, Astronomy and Astrophysics 594 (2016) A97, https://doi.org/10.1051/0004-6361/ 201628980.
23. G. S. Stringfellow and S. E. Woosley, Origin and Distribution of the Elements, ed. G. J. Mathews (Singapore: World Scientific Publishing), 467.

24. B.J. Carr, J.R. Bond and W.D. Arnett, Cosmological consequences of population III stars, The Astrophysical Journal 277 (1984) 445, https: / /adsabs . harvard. edu/pdf/ 1984ApJ...277.445C

25. W.W. Ober, M.F. El Eid and K.J. Fricke, Evolution of massive pregalactic stars, Astronomy and Astrophysics 119 (1983) 61, https: //adsabs.harvard.edu/pdf/ $1983 \mathrm{~A} \div 26 \mathrm{~A} \ldots 119 \ldots 610$

26. J.C. Wheeler, Final evolution of stars in the range $10^{3}-$ $10^{4} \mathrm{M}_{\odot}$, Ap\& SS 50 (1977) 125, https : / / doi.org/10. $1007 / \mathrm{BF} 00648524$.

27. M.F. El Eid and E.R. Hilf, Equation of state for hot an dense n; p; e-mixture with zero charge density, Astronomy and Astrophysics 57 (1977) 243, https://adsabs.harvard. edu/pdf/1977A $\div 26 A . . .57 \ldots 243 \mathrm{E}$

28. G.S. Fraley, Supernovae explosions induced by pair-production instability, The Astrophysical Journal 2 (1968) 96, https: //doi.org/10.1007/BF00651498

29. Z. Barkat, G. Rakavy, and N. Sack, Dynamics of Supernova Explosion Resulting from Pair Formation, PRL 18 (1967) 379, https://doi.org/10.1103/PhysRevLett.18. 379

30. P.J. Montero, H.-T. Janka and E. Müller, Relativistic collapse and explosion of rotating supermassive stars with thermonuclear effects, The Astrophysical Journal 749 (2012) 37, https://doi.org/10.1088/0004-637X/749/ $1 / 37$

31. A. Maeder, Physics, formation and evoiution of rotating stars (Springer science \& Business Media, 2008).

32. C. J. Hansen, S. D. Kawaler, and V. Trimble, in Stellar Interiors (Springer, 2004), pp. 431.

33. S.E. Woosley, A. Heger, and T.A. Weaver, The evolution and explosion of massive stars, Rev. Mod. Phys. 74 (2002) 1015, https://doi.org/10.1103/RevModPhys. 74.1015

34. E. Chatzopoulos and J.C. Wheeler, Effects of rotation on the minimum mass of the primordial progenitors of pair-instability supernovae, The Astrophysical Journal $\mathbf{7 4 8}$ (2012) 42, https://doi.org/10.1088/0004-637X/ $748 / 1 / 42$

35. A. Heger, C.L. Fryer, S.E. Woosley, N. Langer, and D.H. Hartmann, How massive single stars end their life, The Astrophysical Journal 591 (2003) 288, https://doi.org/10. $1086 / 375341$

36. N. Langer, C.A. Norman, A. de Koter, J.S. Vink, M. Cantiello, and S.-C. Yoon, Pair creation supernovae at low and high redshift, Astronomy and Astrophysics 475 (2007) L19, https: //doi.org/10.1051/0004-6361:20078482.

37. A. Kozyreva, S.-C. Yoon, and N. Langer, Explosion and nucleosynthe- sis of low-redshift pair-instability supernovae, Astronomy and Astrophysics 566 (2014) A146, https:// doi.org/10.1051/0004-6361/201423641 
38. J.S. Vink et al., Wind modelling of very massive stars up to 300 solar masses, Astronomy and Astrophysics 531 (2011) A132, https://doi.org/10.1051/0004-6361/ 201116614

39. N. Yusof et al., Evolution and fate of very massive stars, The Astrophysical Journal 433 (2013) 1114, https: / / doi .org/ $10.1093 / \mathrm{mnras} / \mathrm{stt} 794$

40. S.E. Woosley, Pulsational pair-instability supernovae, The Astrophysical Journal 836 (2017) 244, https://doi.org/ $10.3847 / 1538-4357 / 836 / 2 / 244$.

41. A.C. Phillips, The Physics of Stars, (John Wiley \& Sons, 2013).

42. P. Eggenberger et al., The Geneva stellar evolution code, in M.J.P.F.G. Monteiro, Evolution and seismic tools for stellar astrophysics, (Springer, 2008), pp. 43-54.
43. T.A. Weaver, G.B. Zimmerman, and S.E. Woosley, KEPLER: General purpose 1D multizone hydrodynamics code, Astrophysics Source Code Library, ascl:1702.007, https://ui.adsabs.harvard.edu/abs/ 2017ascl.soft $02007 \mathrm{~W}$

44. F.X. Timmes and F.D. Swesty, The Accuracy, Consistency, and Speed of an Electron-Positron Equation of State Based on Table Interpolation of the Helmholtz Free Energy, The Astrophysical Journal Supplement Series 126 (2000) 501, https : //doi.org/10.1086/313304.

45. F.X. Timmes and D. Arnett, The Accuracy, Consistency, and Speed of Five Equations of State for Stellar Hydrodynamics, The Astrophysical Journal Supplement Series 125 (1999) 277, https://doi.org/10.1086/313271 